\title{
The clinical features of polymerase proof-reading associated polyposis (PPAP) and recommendations for patient management
}

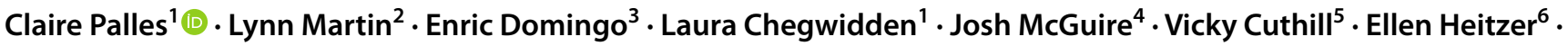 \\ The CORGI Consortium • Rachel Kerr ${ }^{3} \cdot$ David Kerr $^{7} \cdot$ Stephen Kearsey ${ }^{8} \cdot$ Susan K. Clark ${ }^{5,9} \cdot$ Ian Tomlinson ${ }^{2}$. \\ Andrew Latchford ${ }^{5,9}$
}

Received: 14 December 2020 / Accepted: 7 April 2021 / Published online: 5 May 2021

(c) The Author(s) 2021

\begin{abstract}
Pathogenic germline exonuclease domain (ED) variants of POLE and POLDI cause the Mendelian dominant condition polymerase proof-reading associated polyposis (PPAP). We aimed to describe the clinical features of all PPAP patients with probably pathogenic variants. We identified patients with a variants mapping to the EDs of POLE or POLD1 from cancer genetics clinics, a colorectal cancer (CRC) clinical trial, and systematic review of the literature. We used multiple evidence sources to separate ED variants into those with strong evidence of pathogenicity and those of uncertain importance. We performed quantitative analysis of the risk of CRC, colorectal adenomas, endometrial cancer or any cancer in the former group. 132 individuals carried a probably pathogenic ED variant (105 POLE, 27 POLD1). The earliest malignancy was colorectal cancer at 14 . The most common tumour types were colorectal, followed by endometrial in POLD1 heterozygotes and duodenal in POLE heterozygotes. POLD1-mutant cases were at a significantly higher risk of endometrial cancer than POLE heterozygotes. Five individuals with a POLE pathogenic variant, but none with a POLDI pathogenic variant, developed ovarian cancer. Nine patients with $P O L E$ pathogenic variants and one with a $P O L D 1$ pathogenic variant developed brain tumours. Our data provide important evidence for PPAP management. Colonoscopic surveillance is recommended from age 14 and upper-gastrointestinal surveillance from age 25. The management of other tumour risks remains uncertain, but surveillance should be considered. In the absence of strong genotype-phenotype associations, these recommendations should apply to all PPAP patients.
\end{abstract}

Keywords POLE $\cdot$ POLD1 $\cdot$ PPAP $\cdot$ Exonuclease domain mutation

The members of the Infanta Leonor Thrombosis Research Group have been listed in Supplementary.

Claire Palles

c.palles@bham.ac.uk

1 Gastrointestinal Cancer Genetics Laboratory, Institute of Cancer and Genomic Sciences, College of Medical and Dental Sciences, University of Birmingham, Birmingham B15 2TT, UK

2 Edinburgh Cancer Research Centre, Institute of Genetics and Cancer, University of Edinburgh, Crewe Road South, Edinburgh EH4 2XU, UK

3 Department of Oncology, Old Road Campus Research Building, University of Oxford, Roosevelt Drive, Oxford, UK

4 Wellcome Centre for Human Genetics, University of Oxford, Roosevelt Drive, Oxford OX3 7BN, UK
5 Polyposis Registry, St Mark's Hospital, Harrow, London HA1 3UJ, UK

6 Diagnostic and Research Institute of Human Genetics, University of Gratz, Graz, Austria

7 Nuffield Department of Clinical Laboratory Sciences, University of Oxford, John Radcliffe Hospital, Oxford OX3 9DU, UK

8 ZRAB, University of Oxford, 11a Mansfield Road, Oxford OX1 3SZ, UK

9 Department of Surgery and Cancer, Imperial College London, London, UK 


\section{Introduction}

It has historically been thought that Mendelian cancer predisposition syndromes are rarely, if ever, associated with a general predisposition to cancer. This is often perplexing, especially when the genes involved are ubiquitously expressed and/or are involved in fundamental processes such as cell metabolism or DNA repair. In fact, it is increasingly recognised that many of these syndromes confer a smaller, but definitely increased, risk of a wide range of cancers, this having been masked by a combination of early death from specific malignancies and ascertainment bias. Early reports of the spectrum of cancers associated with particular high-penetrance gene defects have often been revised in the light of improved treatment, longer follow-up and identification of gene heterozygotes in extended pedigrees. Almost without exception, these studies have confirmed that the cancers originally described are indeed the major risks, but lesser increased risks of other cancers are also present. Well established examples of the latter include prostate cancer in BRCAI and BRCA2 heterozygotes [1], gastric cancer in individuals with $\mathrm{Li}$ Fraumeni syndrome [2] and head and neck squamous cell cancer, gynaecological squamous cell cancer, oesophageal cancer, and liver, brain, skin and renal tumours in Fanconi anaemia patients [3].

It is therefore premature to report the clinical features of new Mendelian syndromes until several years have elapsed since their identification. This problem is compounded by the fact that the recently identified syndromes inevitably tend to be rarer, so that accumulation of clinical information is slow and it is almost impossible to avoid all ascertainment bias. A recent example of the confusion that can arise is the phenotype of the recessive condition NTHL1associated polyposis (NAP), which initially was based on three families and largely comprises colorectal adenomas and carcinomas [4]; yet, a single NAP patient with seven primary cancers (and multiple non-cancerous tumours) has also been described [5]. Recent papers highlighting the now apparent extended tumour phenotype seen in individuals' with two NTHL1 mutations suggest NTHL1 tumour syndrome as a more accurate name for this condition [6].

In this manuscript, we report the clinical features of a set of families with polymerase proofreading-associated polyposis (PPAP), a Mendelian dominant condition caused by pathogenic variants in the exonuclease domains of POLE and POLD1, the genes encoding the catalytic subunits of DNA polymerases epsilon and delta. We assess the phenotype of those heterozygous for an exonuclease domain (ED) probably pathogenic variant in the context of dysfunction of the proof-reading capability of these genes being expected to cause a 100-fold increase in point mutations in theoretically every dividing cell type in the body. We provide an update of the phenotype of the families when we first described PPAP [7] and combine this with a comprehensive literature review, resulting in a set of guidelines for PPAP management.

\section{Methods}

\section{Patients}

Eligible patients were identified from in-house studies (CORGI [8] - 2349 probands screened in 2013 (includes cases from National Study of Colorectal Cancer Genetics (NSCCG)), 2311 patients screened as part of this study, 1 proband identified as carrier by clinical genetics departments or VICTOR/QUASAR2 [9] - number of patients screened in $2013=1560$, number of additional QUASAR 2 patients screened as part of this study $=287$ ) or a literature review. 48 relatives of probands were screened for the family variant. Most of the cases reported in CORGI and all of the cases in QUASAR 2, were discovered by directly testing for POLE NP_006222.2:p. (Leu424Val) and POLD1 NP_001243778.1:p. (Ser478Asn). One of the new families we report was identified by panel sequencing conducted at the clinical genetics centre that recruited the patient. All individuals with a POLE or POLDI ED variant were included, together with their personal and family histories, including as many details of tumours, screening and other major diseases as were available. For further details see Supplementary Methods.

\section{Variant annotation}

Germline ED variant HGVS descriptions were validated using VariantValidator [10] (Supplementary Table S1) and variants were annotated with conservation scores (PhyloP), in silico predictions of likely impact on protein function (SIFT, POLYPHEN, Grantham) and frequency from gno$\mathrm{mAD}$. Protein sequences of the exonuclease domains of human POLE and POLD1 were aligned using COBALT [11], selecting the identity setting for visualisation and scoring (red indicating full and blue partial conservation). The variants and the exo motifs (regions critical for exonucleolytic catalysis highly conserved between the two enzymes) were mapped onto the alignment (Supplementary Figure S1). We also identified studies that had assessed the function of POLE and POLDI ED variants in model organisms (e.g. Schizosaccharomyces pombe) or in vitro assays. We required an ED variant to fulfil at least one of the following criteria for classification as probably pathogenic: variant cosegregating with disease status in more than four meioses, hypermutation observed in yeast when the equivalent amino 
acid was mutated; or evidence of impaired proof-reading from biochemical assays (Supplementary Figure S2). We also required that where more than one of these was available, the data were compatible and consistent. The remaining ED variants were classified as of unknown significance (Supplementary Information, Supplementary Table S2). Details of the assessments of specific variants are provided in the Supplementary Information. The characteristics of the probably pathogenic variants in terms of frequency in gnomAD, in silico scores and location relative to exo motifs and conserved residues between POLE and POLD1 were also compared to the variants of uncertain pathogenicity and the differences observed inform the recommendations made in the discussion.

\section{Statistical analysis}

Cumulative incidence of CRC, endometrial cancer (EC), colorectal polyps/adenomas and any cancer in probably pathogenic exonuclease domain variant heterozygotes was explored using survival analysis packages survival and survminer in R. Diagnosis of one of the above phenotypes was classed as an event and time of diagnosis was used as the time of event. If no event occurred and current age was known, this information was used to define the follow-up period. If no event occurred, but current age was not known, the last age at any other follow-up (for example, last clinic appointment or last cancer diagnosis) was used for censoring. If age at event was not reported the individual was excluded from the analysis.

\section{Results}

\section{Patient ascertainment}

In 2013, we reported eight PPAP families with germline POLE NP_006222.2:p. (Leu424Val), two families with POLD1 NP_001243778.1:p. (Ser478Asn) and one individual with POLD1 NP_001243778.1:p. (Pro327Leu) [6]. The pathogenic status of these variants has been demonstrated statistically and/or functionally. In this study, we updated and extended the families' pedigrees and clinical details (Supplementary Figure S1, Supplementary Tables S3 and S4). Additional clinical information, including identification of three new heterozygotes, was also obtained for the POLE NP_006222.2:p. (Asn363Lys) family reported by Rohlin et al. [12]. We identified four new independent, unrelated POLE Leu424Val heterozygotes, two from the CORGI study (Fig. 2 Families $\mathrm{M}$ and N, Supplementary Table S3) and two, diagnosed with CRC at ages 46 and 37, in the QUASAR 2 trial (Supplementary Table S3). A total of 36 POLE Leu424Val heterozygotes and 11 of POLD1 Ser478Asn heterozygotes, from 12 and two families respectively, was identified in this way (Supplementary Tables S3 and S4).

Other groups have reported pathogenic germline POLD1 variants NP_001243778.1:p. (Asp316Gly), NP_001243778.1:p. (Asp316His) and NP_001243778.1:p. (Leu474Pro) in patients with colonic polyposis or CRC [13]. These variants co-segregated with affection status in samples from the index case's family members and there is evidence from yeast and biochemical assays that mutation of these residues leads to impaired proof-reading. In our genotyping or sequencing data from 2311 colorectal polyposis or CRC patients (pedigrees $\mathrm{O}$ and $\mathrm{P}$ respectively in Fig. 2, clinical features in Supplementary Table S3) we identified NP_001243778.1:p. (Asp316Asn) in one family (two heterozygous individuals) and Leu474Pro in another (two heterozygous individuals). In CORGI $1 \%$ of patients screened were found to carry POLE Leu424Val, POLD1 Ser478Asn, POLD1 Asp316Asn or POLD1 Leu474Pro and in QUASAR 2/VICTOR clinical trials $0.1 \%$ of CRC patients screened carried POLE Leu424Val or POLD1 Ser478Asn.

We performed a literature review to identify other individuals heterozygous for potentially pathogenic germline $P O L E$ and POLD1 ED variants. Using criteria described in the Methods and Supplementary Information, we identified "probably pathogenic" $P O L E$ and $P O L D 1$ germline ED variants - constitutional variants with at least one additional data type supporting their disease-causing effects - in 69 and 11 patients respectively (Fig. 1, Table 1, Supplementary Tables S3 and S4). Information on the 10 POLE and 9 $P O L D 1$ variants identified from the literature, which did not meet our criteria for "probably pathogenic", can be found in Supplementary Table S2. Where available age at diagnosis and adenoma burden is indicated for each affected individual. + indicates an individual heterozygous genotype for the variant indicated, - indicates an individual who underwent genotyping but was negative.

\section{Tumour spectrum in PPAP}

Individuals heterozygous for ED variants with probable or greater likelihood of being pathogenic (105 POLE, 27 $P O L D 1)$ were included in the clinical phenotype assessment (Table 2, Supplementary Tables S3 and S4). Sex was available for 94 individuals.

\section{Colorectal tumours}

One hundred (95\%) POLE variant heterozygotes had a colorectal tumour phenotype (adenomas and/or carcinoma). The median ages at diagnosis of CRC and polyps were 44.5 and 36 respectively. Polyp burden was available for 59/78 POLE ED variant heterozygotes with polyps and ranged from one 
Family M:

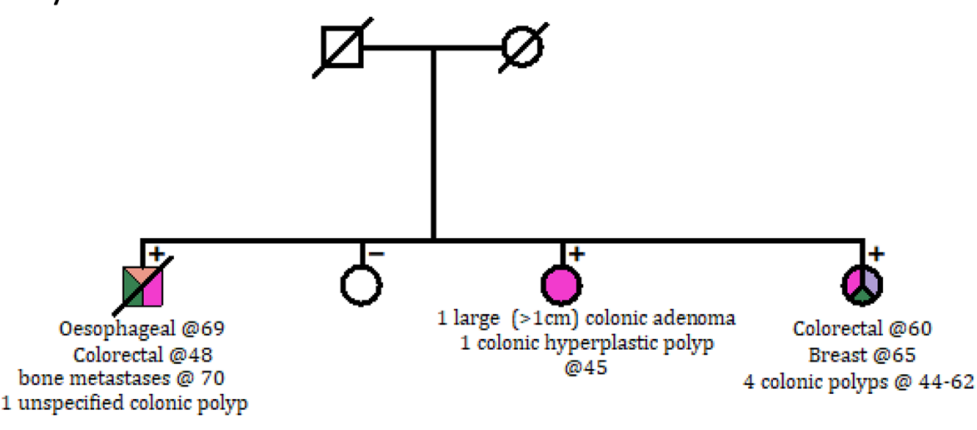

Family O:

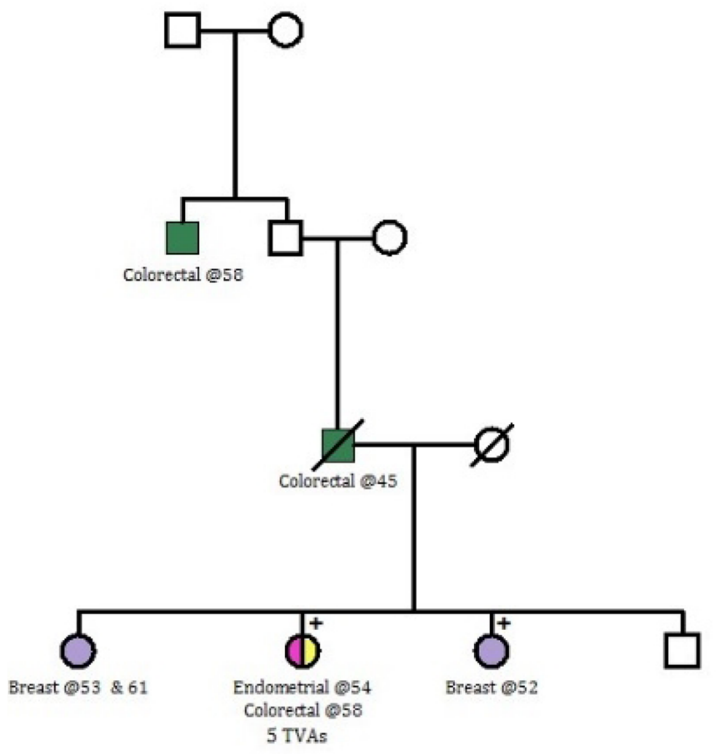

Family $\mathrm{P}$ :

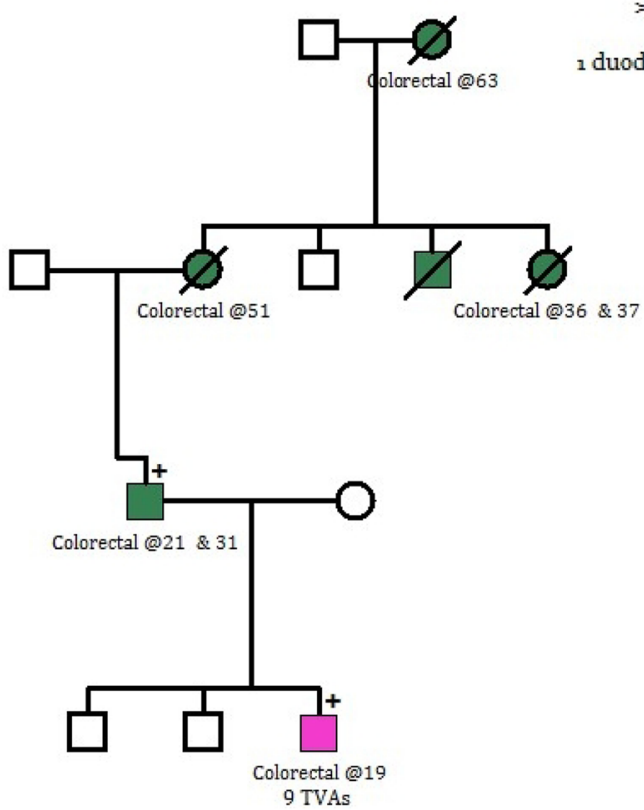

Family N:
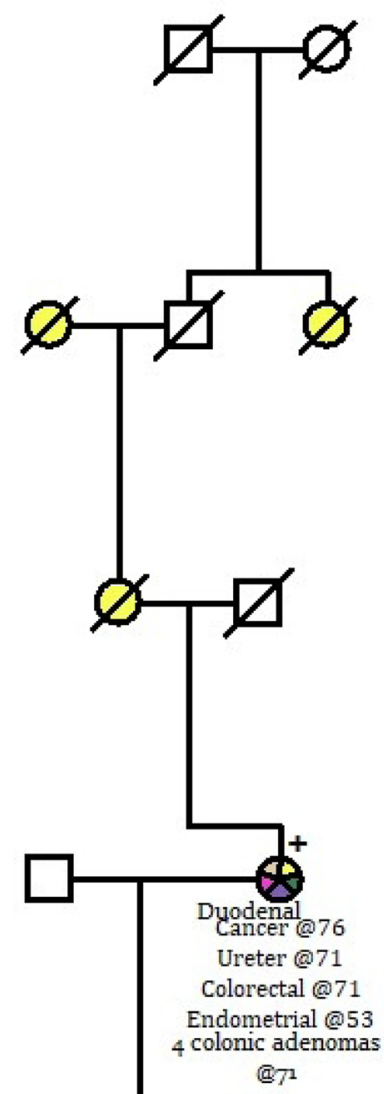

Colorectal@46 10 colonic adenomas @ $46-51$

adenoma $(>1 \mathrm{~cm})$ (9) 51

Definitions

$\square$ Endometrial cancer

- Colorectal adenomas

$\square$ Ureter cancer

$\square$ Colorectal cancer

$\square$ skin cancer, basal cell

Cancer of the small intestine

$\square$ Oesophageal cancer

Fig. 1 Pedigrees of the families in which new carriers of POLE and POLD1 ED probably pathogenic variants were found 


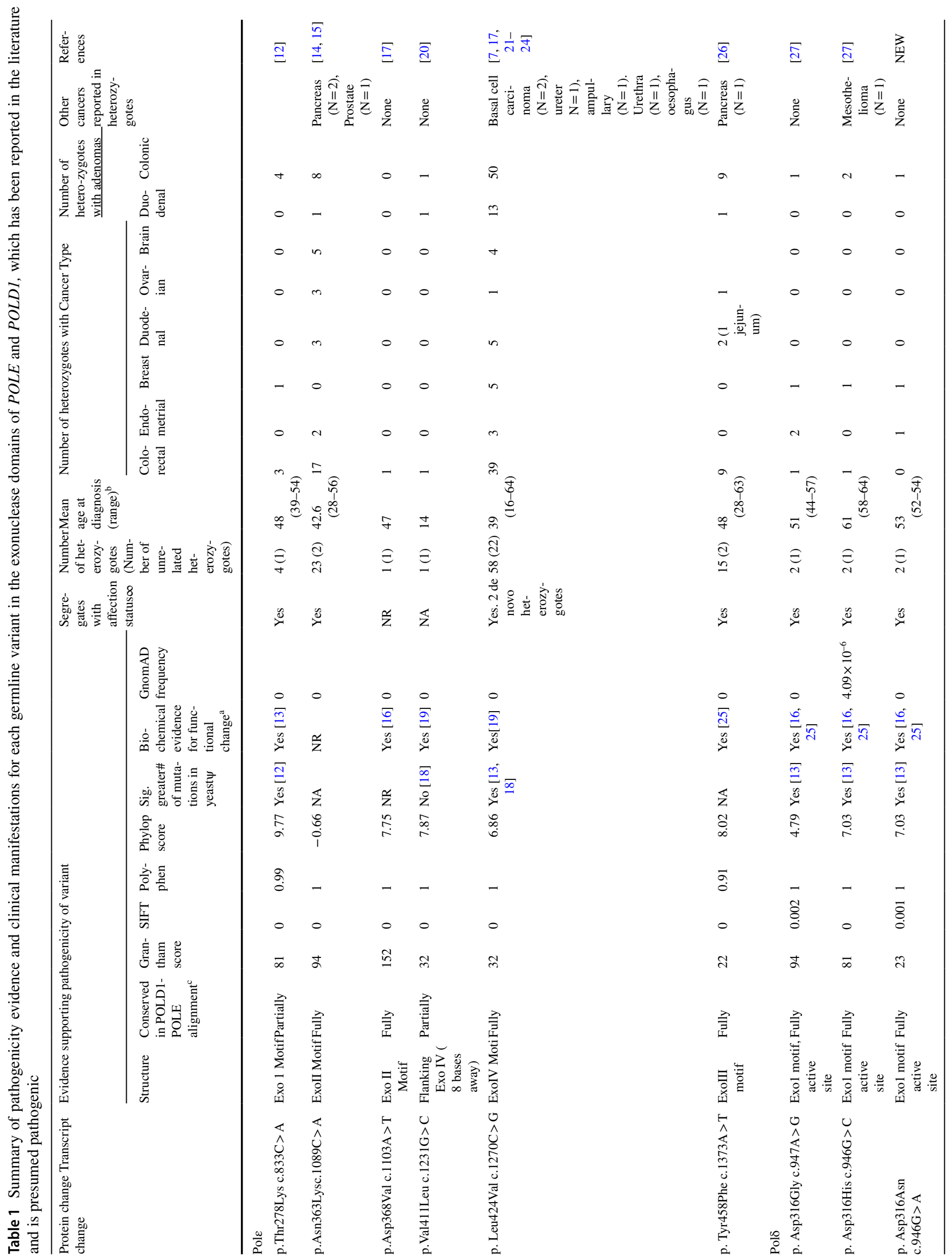




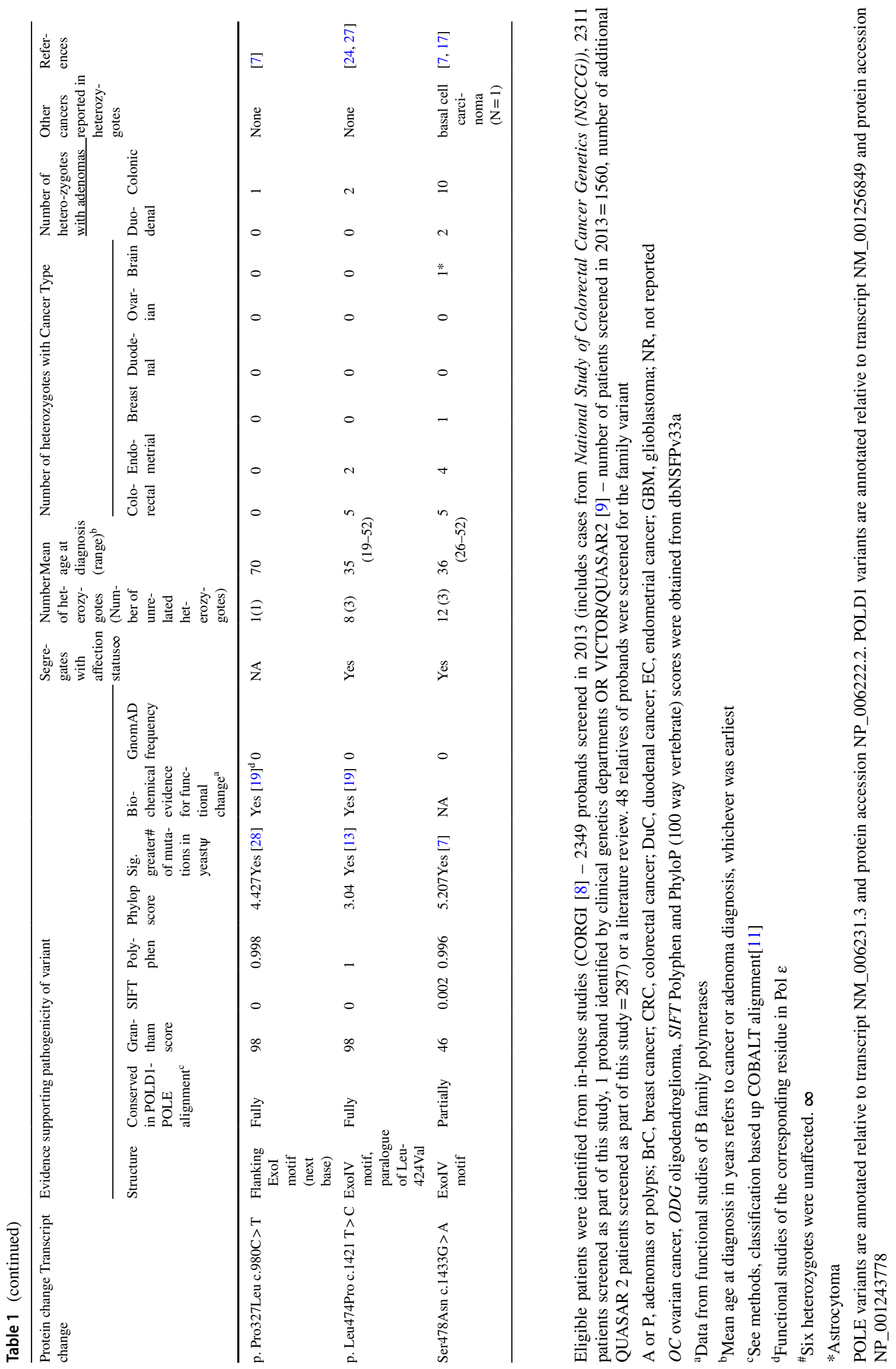


Table 2 Summary information on polyp and cancer phenotypes observed in heterozygotes of likely pathogenic ED variants

Gene Number of heterozygotes with specified cancer type (Median age at diagnosis)

Number of heterozygotes with adenomas (Median age at diagnosis)

\begin{tabular}{lllllllll}
\cline { 2 - 6 } & Colorectal & Endometrial & Breast & Duodenal & Ovarian & Brain & Duodenal & Colonic \\
\hline POLE & $74 / 105(44.5)$ & $5 / 43(53)$ & $6 / 43(49)$ & $10 / 105(54)$ & $5 / 43(45)$ & $9 / 105(35)$ & $16 / 105(51)$ & $78 / 105(36)$ \\
POLD1 & $12 / 27(41)$ & $9 / 17(52)$ & $4 / 17(62)$ & $0 / 17(\mathrm{NA})$ & $0 / 17(\mathrm{NA})$ & $1 / 27(26)$ & $2 / 27(55)$ & $18 / 27(43)$ \\
\hline
\end{tabular}

Metastatic cancers were excluded from this summary

For endometrial, breast and ovarian cancers, counts in female heterozygotes are displayed

to 100 , with a median of 12 . Twenty-three of the 27 POLD1 heterozygotes had a colorectal tumour phenotype. The median ages at diagnosis of CRC and polyps were 41 and 43 years respectively. Polyp burden was available for 17/18 heterozygotes with polyps and ranged from two to 70 , with a median of 13 .

Seventy of the 105 POLE variant heterozygotes developed CRC, at presentation or during follow-up (median age of diagnosis 43). 48 of these also had reported colorectal adenomas (CRAs). Age at diagnosis of CRAs was available for 40 of these patients; 10 patients had a history of adenoma removal prior to cancer diagnosis with the remained being diagnosed with adenomas synchronously with CRC or at a later time. Twelve of 27 (44\%) POLD1 variant heterozygotes developed CRC, none had prior adenoma removal and 5 had no reports of CRAs. The difference in frequency of CRCs and adenomas was not significantly different between POLE and $P O L D 1$ heterozygotes (Fisher's exact test, $\mathrm{CRC} \mathrm{p}=0.12$, CRA $\mathrm{p}=0.19$ ).

Microsatellite instability (MSI) status was available for 29 colorectal tumours from POLE variant heterozygotes and three from $P O L D 1$ variant heterozygotes; four (all carcinomas from POLE variant heterozygotes) of $28 \mathrm{CRCs}$ (14.3\%) and no adenomas were MSI + . Loss of mismatch repair (MMR) proteins (assessed by immunohistochemistry) was reported in CRCs from three of the four MSI cases. No information regarding MMR protein loss was available for the fourth case, just the number of microsatellites that were unstable.

Most colorectal polyps were tubular or tubulovillous adenomas, although nine POLE and five POLD1 variant heterozygotes also had hyperplastic polyps (Supplementary Tables S3 and S4). Sixteen variant heterozygotes were diagnosed with multiple CRCs. Ten of these were synchronous cancers involving 2-4 tumours and in the remainder, CRC developed 1-22 years later (median 10 years). Those developing multiple CRCs carried POLE Leu424Val $(\mathrm{N}=10)$, POLE Thr278Lys $(\mathrm{N}=2)$, POLE Asn363Lys $(\mathrm{N}=2)$, POLD1 Ser478Asn ( $=1)$, POLD1 Leu474Pro $(\mathrm{N}=1)$.

Information on surgery was available for 24 participants, of whom 19 underwent colectomy (4 segmental and 15 extended) following CRC diagnosis. Based on available information, in three cases colectomy (reported as one panproctocolectomy, one extended left hemi-colectomy and one right hemi-colectomy) was chosen following metachronic cancer and a further four participants underwent prophylactic surgery following diagnosis of multiple bowel adenomas (one proctocolectomy, one subtotal and one total colectomy; one underwent "colectomy" but further details of this surgery are not available).

All CORGI participants with probably pathogenic ED variant were undergoing colonoscopic surveillance; one developed cancer on surveillance and one developed cancer after having been discharged from follow-up.

\section{Upper gastrointestinal tumours}

After colorectal tumours, duodenal tumours were the next most frequent lesions in POLE variant heterozygotes. Ten of 105 (9.5\%) probably pathogenic POLE ED variant heterozygotes developed duodenal cancer (DC; median age 55) and $16(15 \%)$ developed one or more duodenal adenomas (DAs; median age 43). 23 probably pathogenic POLE ED variant heterozygotes developed both a colorectal phenotype (CRC and or CRAs) and a duodenal phenotype (DC and or DAs). In two cases, DAs arose before age 18. No DCs have arisen to date in POLD1 ED heterozygotes, but DAs were diagnosed in $2 / 27$ cases ( $9 \%$; median age 55 ) who also had CRC and or CRAs. The difference in frequency of DC and DA was not significant (Fisher's exact test DC $p=0.21$, DA $\mathrm{p}=0.36$ ).

\section{Endometrial and ovarian cancers}

EC was the most common malignancy in female POLD1 variant heterozygotes $(9 / 17,53 \%)$, with three diagnosed under the age of 50.5/43 POLE variant heterozygotes (12\%) were diagnosed with EC, one before the age of 50. The frequency of EC was significantly lower in POLE variant heterozygotes (Fisher's exact test, $\mathrm{p}=0.001$ ) (Fig. 2a). Five female POLE ED variant heterozygotes 

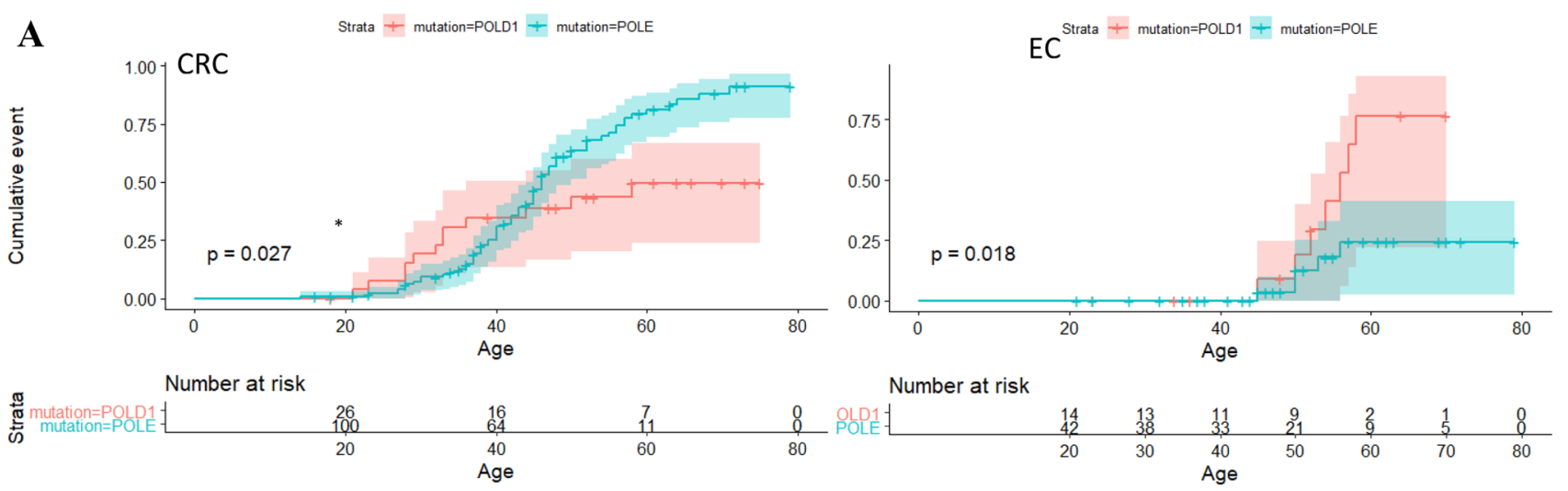

Number at risk
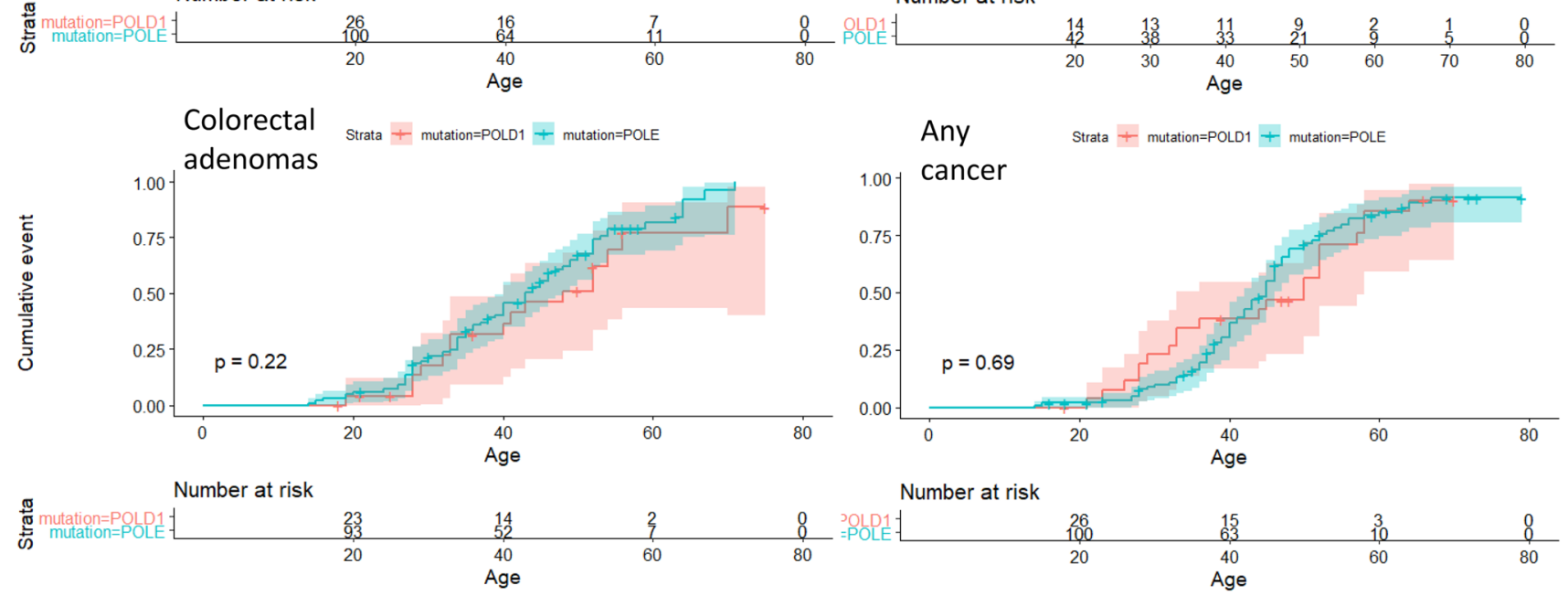

Number at risk

B

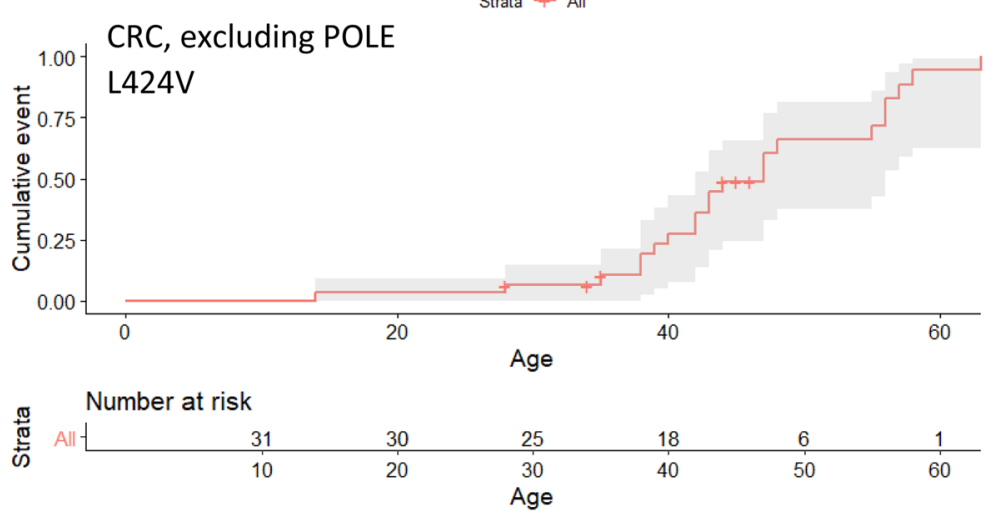

Fig. 2 Graphs showing the cumulative risk of developing CRC, colorectal adenomas, endometrial cancer and any cancer in carriers of probably pathogenic variants in POLE and POLDI

developed ovarian cancer (OC) between the ages of 33 and 45 , including one with bilateral disease age 40 years, but no OCs were found in POLDI ED variant heterozygotes (Fisher's exact test, $\mathrm{p}=0.31$ )). Two patients were diagnosed with OC at the same time as their EC, although it was not clear whether the synchronous lesions had independent origins. No further details on the histology or molecular biology of the ovarian cancers were available. Data were also incomplete with respect to premalignant gynaecological lesions. However, two POLE Leu424Val heterozygotes underwent hysterectomy for benign pathology, specifically endometrial dysplasia or hyperplasia, or endometriotic cysts.

Out of a total of 105 POLE carriers and 27 POLD1 carriers, of which 43 POLE carriers and 17 POLD1 carriers were female, the following had sufficient information to be included in the analysis of cumulative risk of (1) CRC: POLE $\mathrm{N}=103$ POLD1 N=27 (2) EC POLE $N=43$, POLD1 $\mathrm{N}=13$, (3) colorectal adenomas POLE $\mathrm{N}=87$, POLD1 $\mathrm{N}=25$ (4) any cancer POLE N=94 POLD1 N=27. a Gene stratified analysis: Male and female heterozygotes were analysed together apart from in the EC plot which was generated 
using data from female heterozygotes only. "Any cancer" includes brain tumours but not duodenal or colorectal adenomas. No correction was made for screening or surgical interventions. b CRC cumulative risk in POLE presumed pathogenic variant heterozygotes, excluding POLE Leu424Val. Shaded areas represent $95 \%$ confidence intervals *P-value remains significant when POLE Leu424Val heterozygotes are excluded, $\mathrm{p}=0.018$.

\section{Brain tumours}

Brain tumours (two astrocytomas at age 26) were observed in one of the first POLD1 Ser478Asn heterozygotes described. Brain tumours have also now been reported in nine of 105 (9\%) of POLE variant heterozygotes. Two POLE Leu424Val heterozygotes developed glioblastomas at age 47 and age 61 respectively, one Leu424Val heterozygote developed an astrocytoma at age 15 and one Leu424Val heterozygote developed an oligodendroglioma aged 30. Five Asn363Lys heterozygotes developed primary brain tumours, comprising four glioblastomas diagnosed between ages 30 and 52, and one of unspecified type at 35 .

\section{Other common tumours}

Breast cancer was not described in the original PPAP families, but there are now multiple reports of breast cancer in female patients with PPAP. Six of 43 (14\%) female POLE variant heterozygotes developed breast cancer (median age 49 years, range $38-65)$, as did 4/17 (24\%) female POLDI variant heterozygotes (median age 62 years, range 52-65). The latter frequency is clearly higher than the general population. Despite some recent reports that POLE and POLDI variants predispose to prostate cancer [29], only one patient developed prostate cancer (aged 51) in the families analysed.

\section{Multiple tumours}

15/51 (29\%) male and 20/43 (46.5\%) female POLE ED variant heterozygotes developed benign or malignant tumours of more than one site to the date of follow-up. For POLD1, the equivalent frequencies were $2 / 10$ (20\%) males and 10/17 (59\%) females. Three out of 132 patients had synchronous tumours of different organs.
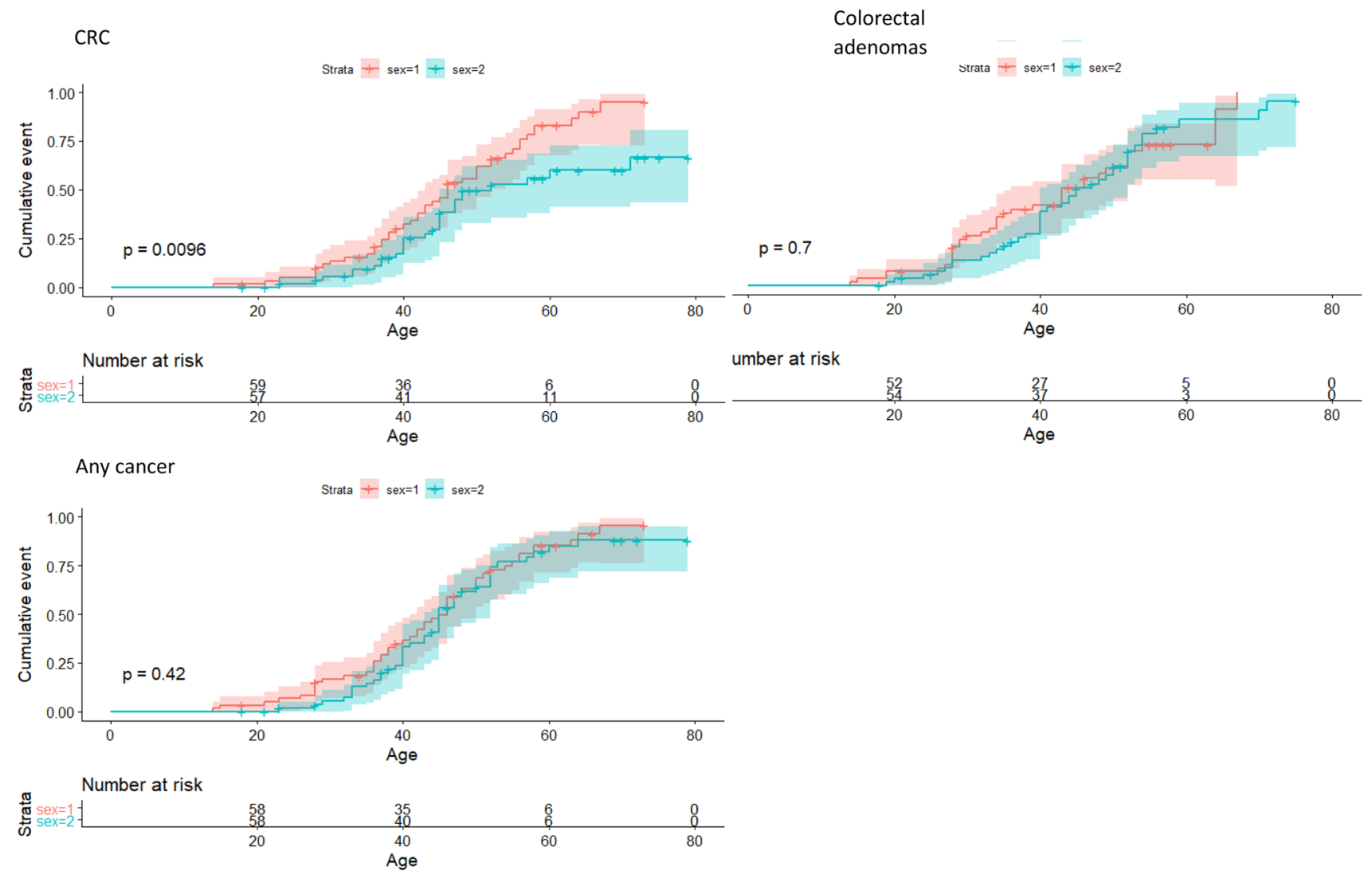

Fig. 3 Graphs showing the cumulative risk of developing CRC, colorectal adenomas and any cancer in male and female carriers of probably pathogenic variants in POLE and POLD1. POLD1 and POLE
ED variant heterozygotes have been grouped together. Shaded areas represent $95 \%$ confidence intervals 


\section{Penetrance of probably pathogenic variants}

Cumulative incidence curves (Fig. 2a) show the age-dependent penetrance of the following phenotypes in those heterozygous for a probably pathogenic POLE and POLDI ED variant: CRC; colorectal adenoma(s); EC; and any cancer including brain tumour. 15 patients developed CRC before age 30 . The risk of CRC by age 70 years was approximately $90 \%$ for POLE variant heterozygotes and lower (around $50 \%)$ for POLD1 ED variant heterozygotes $(\mathrm{p}=0.027)$. Conversely, $P O L D 1$ ED variant heterozygotes had a higher risk over time than POLE ED variant heterozygotes of developing $\mathrm{EC}(\mathrm{P}=0.018)$. The risk of $\mathrm{EC}$ by age 70 was in the region of $75 \%$ for $P O L D 1$ and about $25 \%$ for POLE variant heterozygotes. No ECs developed before age 45 years in any of these variant heterozygotes. Males had a higher lifetime risk of CRC than females $(\mathrm{P}=0.0096$; Fig. 3) for both genes, although this association was not present for colorectal adenomas or when cancers of any organ were considered (Fig. 3). We compared CRC incidence between those heterozygous for the prototypic Leu424Val variant and other variants, but no clear difference was found (Fig. 2b).

\section{Discussion}

In this study, we have provided a comprehensive description of the clinicopathological features of PPAP in those heterozygous for POLE and POLD1 ED variants with supporting evidence of a pathogenic impact. The cumulative incidence of CRC in POLE and POLDI variant heterozygotes is estimated at approximately $90 \%$ and $50 \%$ respectively. These estimates are higher than previous reports [30], probably because of the exclusion of variants of uncertain pathogenicity and the variable mixture between studies of PPAP patients who were screened because of family history and others whose first cancer occurred before surveillance began. Most patients have an attenuated polyposis phenotype (10-100 polyps), with greater polyp numbers in a few cases, but a minority of cases have a Lynch syndrome-like phenotype with early-onset CRC and few polyps. Individuals without CRAs were either young (aged 24 and 18 respectively), or carried a relatively rare probably pathogenic variant and presented with an extra-colonic tumour (POLE p.Tyr458Phe, $P O L D 1$ p. Asp316Gly, POLD1 p. Asp316Asn, POLD1 p. Leu474Pro).

We have expanded the spectrum of intestinal cancers to include benign and malignant duodenal tumours. $19 \%$ of all probably pathogenic ED variant heterozygotes had both a colorectal and a duodenal phenotype. Unfortunately, phenotypic data regarding the upper GI tract are somewhat limited and indeed many of the patients reported in the literature are likely not to have undergone upper GI endoscopy at all. When only those who have had an upper GI endoscopy were included, DAs were seen in over $50 \%$ of $\mathrm{ED}$ variant heterozygotes.

$\mathrm{EC}$ is the most common extra-intestinal malignancy in PPAP, with lower risks than CRC. The risk is higher in POLDl heterozygotes. OC is also relatively frequent, sometimes synchronous with EC, although no consistent assessment of histology or clonal origins was possible. There is also growing evidence that a variety of brain tumours can occur as part of PPAP.

Caution is advised in interpreting the details of some of our findings, including the quantitative cancer risk estimates and the intriguing possibility of genotype-phenotype correlations. Potential problems include the small numbers of affected individuals from a limited number of families, the variable extent of cancer screening, and ascertainment bias for genetic testing. Such issues are unavoidable for rare conditions, and must be take into account wherever possible in the clinical setting.

How do our data inform clinical management? Colorectal surveillance is clearly advisable. Bellido et al. [27] suggested colonoscopy from the age of 18 years. Given that we identified an individual with a CRA aged 15 and Wimmer et al. [20] identified a CRC in a 14 year old, we suggest that earlier colonoscopic assessment at age 14 should be performed in all PPAP patients. It is noteworthy that $26 / 86$ (30\%) CRC cases were in patients who did not have adenomas and no clear correlation between polyp burden and cancer risk has been demonstrated. Furthermore, although the tumours we originally described in POLE and POLDI carriers were microsatellite-stable, the frequency of MSI (12.5\%) that we now describe in CRCs is similar to that in sporadic CRCs, despite the earlier onset of the former. Whether MSI in PPAP CRC results from somatic loss of mismatch repair or an unappreciated direct effect of constitutive proofreading deficiency, it could result in accelerated progression to cancer. Given the above uncertainties, we recommend at least biennial colonoscopy, compared with 1-2 yearly suggested by Bellido et al. [27]. Prophylactic surgical intervention may be appropriate for PPAP cases with a severe polyp phenotype: our data suggest that colectomy and ileo-rectal or ileo-distal sigmoid anastomosis would usually be most appropriate. Surgery for those with PPAP in whom CRC has already arisen needs to be individualised and no strong recommendation can be given; factors including phenotype, expected functional outcome and the metachronous cancer risk all need to be considered.

The finding of frequent DAs and DC suggests that routine upper gastrointestinal tract surveillance would be beneficial. In the absence of robust data, we suggest that surveillance is based on the system advocated for FAP [31], starting at age 25 . The role of duodenal polypectomy is unproven, even in 
FAP, but it would seem reasonable to consider polypectomy in PPAP when adenomas reach $1 \mathrm{~cm}$ in size.

Endometrial cancer is common in PPAP and it is logical to think that gynaecological surveillance would be warranted. Indeed, Bellido and colleagues [27] proposed endometrial surveillance in patients with $P O L D 1$ pathogenic variants, commencing age 40 years. However, there are no robust data to support gynaecological screening in Lynch syndrome, which has a comparable or higher risk of gynaecological cancer, even though it is performed in some centres. Instead of gynaecological surveillance, Lynch syndrome patients are usually counselled regarding the possibility of risk-reducing surgery when they have completed their family [32]. For POLE carriers, given the finding of 5 cases with ovarian cancer, this counselling should include a discussion of both risk reducing hysterectomy and bilateral salpingo-oophorectomy (BSO). We suggest a similar approach for women with PPAP, since the EC risk is appreciable with either POLD1 or POLE ED variant heterozygotes.

For the other cancers observed in PPAP families, the absolute risk appears relatively small and below the threshold at which routine surveillance would be recommended. The lifetime risk of breast cancer in the female UK population is $12 \%$ and the risk of developing breast cancer in the next 10 years if aged 50 is $3.54 \%$ whereas the risk of breast cancer by age 60 is approximately $30 \%$ in POLD1 and $20 \%$ in POLE ED pathogenic variant heterozygotes included in our analysis (Supplementary Figure S4). Whilst this is based on only 40 female POLE ED heterozygotes and 17 female POLD 1 heterozygotes, we conclude that female POLDl ED heterozygotes in particular may be at an increased risk of developing breast cancer compared to the general population, but this is not proven. Specifically, there is, as yet, insufficient evidence that the risk observed is high enough to fulfil the criteria for moderate risk breast screening according to UK NICE (Familial breast cancer: classification, care and managing breast cancer and related risks in people with a family history of breast cancer, Clinical guideline [CG164] updated: March 2017 [33]). We suggest $P O L E$ and POLDI ED heterozygotes could be referred to a high risk breast screening service for discussion. The lack of data about type of breast cancer, hormone receptor status and the inherent bias in our cohort make it difficult to give robust recommendations. Discussion in a specialised unit is sensible particularly where there is clustering of breast cancer cases or the presence of very young onset breast cancer in a first degree relative. Certainly, breast awareness and self-examination should be encouraged in all female patients with PPAP. Whilst there are no data to support that it is effective, increasing awareness and empowering patients to be involved in the management of their condition should be supported.
The PPAP phenotype is in some ways a hybrid between attenuated adenomatous polyposis (as seen in other DNA repair deficiency syndromes such as MAP) and Lynch syndrome, especially as regards the high EC risk. CRC and EC cancer patients with somatic POLE ED mutations have been shown to have a favourable prognosis $[34,35]$ and it would be interesting in future studies to investigate whether the prognosis of cancer patients with germline POLE or POLD1 mutations is also better. There is currently no consensus as to who should have diagnostic testing for PPAP (screening of EDs of POLE and $P O L D 1$ for pathogenic variants); we suggest that it should be considered for those with unexplained adenomatous polyposis, as well as those with a family history fulfilling the Amsterdam or modified Bethesda criteria, especially if no pathogenic mismatch repair variants have been found. Distinguishing pathogenic, PPAP-causing variants from non-pathogenic variants in POLE and POLDI may be challenging. Based on the features of the mutations described here and the likely absence of functional data to help assessment of novel mutations in the clinic, we suggest applying the following filtering steps to distinguish a likely pathogenic variant:

(1) Maps to the exonuclease domain of POLE (amino acids 268-471) or POLD1 (amino acids 304-533)

(2) Allele frequency of $<1 \times 10^{-5}$ in non-Finnish European gnomAD data

(3) Maps to or flanks an exo motif and affects an amino acid that is perfectly or highly conserved in a POLEPOLD1 protein alignment

(4) Classed as pathogenic by two or more in silico tools

(5) Results in a protein predicted to retain polymerase and regulatory functions.

Additional DNA samples from relatives for co-segregation studies, and from tumours for mutation burden, spectrum and signature analysis are also very helpful. It remains desirable to find an in vitro system for rapid testing of novel variants. Design of functional assays in accordance with American College of Medical Genetics and Genomics (ACMG) and the Association for Molecular Pathology (AMP) guidelines would aid greatly with variant interpretation [36]. The set of variants selected here as probably pathogenic all had supporting evidence from at least one of the following: co-segregation studies, mutator phenotype assessment in yeast assays or biochemical proof reading assays, in addition to fulfilling the five criteria above. If any of these supporting lines of evidence were conflicting or inconclusive, the variant was classed as being of unknown significance (e.g. POLE W347C and POLE L460M). It is possible that we have excluded variants that are pathogenic, but we decided that strict classification criteria for pathogenic 
variants was required in order to give an accurate survey of the clinical features of those with PPAP.

This manuscript is a step forward in describing the clinical features of PPAP based on variants with strong evidence of pathogenicity with implications for diagnostic testing algorithms and hence screening. With time, the understanding of this condition and its cancer risk will no doubt evolve and inform updates to our recommendations for clinical management. We highlight the difficulties in identifying truly pathogenic variants and provide suggestions that could be incorporated in a more formal variant classification system for suspected PPAP-causing variants.

Supplementary Information The online version contains supplementary material available at https://doi.org/10.1007/s10689-021-00256-y.

Acknowledgements We would like to thank all of the clinicians and healthcare providers who have assisted in recruiting patients to CORGI and CORGI 2.0 and obtaining samples and clinical information. We would also like to thank all of the patients who have consented to use of their samples and medical information for the purposes of this study. We also thank Margareta Nordling, Department of Medical Genetics, University of Gothenburg, Sweden for updated information on the family with POLE Asn363Lys heterozygotes. IT acknowledges Cancer Research UK Programme Grant and ERC EVOCAN project funding. $\mathrm{CP}$ acknowledges Bowel Cancer UK funding. We acknowledge the networking support of the COST Actions BM1206 and CA17118.

Author Contributions Conceptualization and Supervision: AL and IT. Data curation: CP, AL, VC. Formal Analysis: CP. Investigation: CP, LM, LC, ED, JM, EH, SK and The CORGI Consortium. CP and VC performed the literature review. Resources: IT, DK and RK and DK. Writing - original draft: IT, AL, CP. Writing - review \& editing: IT, AL, CP, SC, EH, SK, BD (CORGI Consortium), GE (CORGI Consortium).

Data Availability Individual level information for all patients identified during this research can be found in the supplementary tables.

\section{Compliance with ethical standards}

Conflict of interest None of the authors report any conflict of interest.

Ethical approval CORGI and CORGI2 have been approved by South Central Hampshire - A Research Ethics Research Ethics Committee and South Central Oxford A Research Committee, references 17/ SC/0079, 06/Q1702/9. QUASAR 2 was approved by West Midlands Research Ethics Committee (Edgbaston, Birmingham, UK; REC reference: 04/MRE/11/18. We have received and archived written consent for participation/publication from every individual whose data is included from the CORGI, CORGI 2 and QUASAR 2 clinical trial. All other individual data is taken from published manuscripts which are referenced in the manuscript.

Open Access This article is licensed under a Creative Commons Attribution 4.0 International License, which permits use, sharing, adaptation, distribution and reproduction in any medium or format, as long as you give appropriate credit to the original author(s) and the source, provide a link to the Creative Commons licence, and indicate if changes were made. The images or other third party material in this article are included in the article's Creative Commons licence, unless indicated otherwise in a credit line to the material. If material is not included in the article's Creative Commons licence and your intended use is not permitted by statutory regulation or exceeds the permitted use, you will need to obtain permission directly from the copyright holder. To view a copy of this licence, visit http://creativecommons.org/licenses/by/4.0/.

\section{References}

1. Kote-Jarai $\mathrm{Z}$ et al (2011) BRCA2 is a moderate penetrance gene contributing to young-onset prostate cancer: implications for genetic testing in prostate cancer patients. Br J Cancer 105(8):1230-1234

2. Masciari S et al (2011) Gastric cancer in individuals with LiFraumeni syndrome. Genet Med 13(7):651-657

3. D'Andrea DAAMD (2010) The fanconi anemia and breast cancer susceptibility pathways. New England J Med 362(20):1909-1919

4. Weren RD et al (2015) A germline homozygous mutation in the base-excision repair gene NTHL1 causes adenomatous polyposis and colorectal cancer. Nat Genet 47(6):668-671

5. Rivera B et al (2015) Biallelic NTHL1 Mutations in a Woman with Multiple Primary Tumors. N Engl J Med 373(20):1985-1986

6. Kuiper, R.P., et al., NTHL1 Tumor Syndrome, in GeneReviews $((R))$, M.P. Adam, et al., Editors. 2020: Seattle (WA).

7. Palles $\mathrm{C}$ et al (2013) Germline mutations affecting the proofreading domains of POLE and POLD1 predispose to colorectal adenomas and carcinomas. Nat Genet 45(2):136-144

8. Tomlinson I et al (2007) A genome-wide association scan of tag SNPs identifies a susceptibility variant for colorectal cancer at 8q24.21. Nat Genet 39(8):984-988

9. Kerr RS et al (2016) Adjuvant capecitabine plus bevacizumab versus capecitabine alone in patients with colorectal cancer (QUASAR 2): an open-label, randomised phase 3 trial. Lancet Oncol 17(11):1543-1557

10. Freeman PJ et al (2018) VariantValidator: accurate validation, mapping, and formatting of sequence variation descriptions. Hum Mutat 39(1):61-68

11. Papadopoulos JS, Agarwala R (2007) COBALT: constraint-based alignment tool for multiple protein sequences. Bioinformatics 23(9):1073-1079

12. Castellsague $E$ et al (2019) Novel POLE pathogenic germline variant in a family with multiple primary tumors results in distinct mutational signatures. Hum Mutat 40(1):36-41

13. Murphy K et al (2006) A method to select for mutator DNA polymerase deltas in Saccharomyces cerevisiae. Genome 49(4):403-410

14. Rohlin A et al (2014) A mutation in POLE predisposing to a multi-tumour phenotype. Int J Oncol 45(1):77-81

15. Vande PP et al (2019) Germline mutation pN.363K in POLE is associated with an increased risk of colorectal cancer and giant cell glioblastoma. Fam Cancer 18(2):173-178

16. Abdus Sattar AK et al (1996) Functional consequences and exonuclease kinetic parameters of point mutations in bacteriophage T4 DNA polymerase. Biochemistry 35(51):16621-16629

17. Chubb D et al (2015) Genetic diagnosis of high-penetrance susceptibility for colorectal cancer (CRC) is achievable for a high proportion of familial CRC by exome sequencing. J Clin Oncol 33(5):426-432

18. Barbari SR et al. (2018) Functional Analysis of Cancer Associated DNA Polymerase epsilon Variants in Saccharomyces cerevisiae. G3 Bethesda 8(3): 1019-1029.

19. Shinbrot E et al (2014) Exonuclease mutations in DNA polymerase epsilon reveal replication strand specific mutation patterns and human origins of replication. Genome Res 24(11):1740-1750 
20. Wimmer K et al (2017) A novel germline POLE mutation causes an early onset cancer prone syndrome mimicking constitutional mismatch repair deficiency. Fam Cancer 16(1):67-71

21. Elsayed FA et al (2015) Germline variants in POLE are associated with early onset mismatch repair deficient colorectal cancer. Eur J Hum Genet 23(8):1080-1084

22. Rohlin A et al (2016) GREM1 and POLE variants in hereditary colorectal cancer syndromes. Genes Chromosomes Cancer 55(1):95-106

23. Spier I et al (2015) Frequency and phenotypic spectrum of germline mutations in POLE and seven other polymerase genes in 266 patients with colorectal adenomas and carcinomas. Int J Cancer 137(2):320-331

24. Valle L et al (2014) New insights into POLE and POLD1 germline mutations in familial colorectal cancer and polyposis. Hum Mol Genet 23(13):3506-3512

25. Derbyshire V, Grindley ND, Joyce CM (1991) The 3'-5' exonuclease of DNA polymerase I of Escherichia coli: contribution of each amino acid at the active site to the reaction. EMBO J 10(1):17-24

26. Hansen MF et al (2015) A novel POLE mutation associated with cancers of colon, pancreas, ovaries and small intestine. Fam Cancer 14(3):437-448

27. Bellido $\mathrm{F}$ et al (2016) POLE and POLD1 mutations in 529 kindred with familial colorectal cancer and/or polyposis: review of reported cases and recommendations for genetic testing and surveillance. Genet Med 18(4):325-332

28. Kane DP, Shcherbakova PV (2014) A common cancer-associated DNA polymerase epsilon mutation causes an exceptionally strong mutator phenotype, indicating fidelity defects distinct from loss of proofreading. Cancer Res 74(7):1895-1901
29. Leongamornlert DA et al (2019) Germline DNA repair gene mutations in young-onset prostate cancer cases in the UK: evidence for a more extensive genetic panel. Eur Urol 76(3):329-337

30. Buchanan, D.D., et al., Risk of colorectal cancer for carriers of a germ-line mutation in POLE or POLD1. Genet Med, 2017.

31. Vasen HF et al (2008) Guidelines for the clinical management of familial adenomatous polyposis (FAP). Gut 57(5):704-713

32. Crosbie EJ et al (2019) The Manchester International Consensus Group recommendations for the management of gynecological cancers in Lynch syndrome. Genet Med 21(10):2390-2400

33. Familial breast cancer: classification, care and managing breast cancer and related risks in people with a family history of breast cancer, Clinical guideline [CG164] updated: March 2017.

34. Domingo E et al (2016) Somatic POLE proofreading domain mutation, immune response, and prognosis in colorectal cancer: a retrospective, pooled biomarker study. Lancet Gastroenterol Hepatol 1(3):207-216

35. van Gool IC, Bosse T, Church DN (2016) POLE proofreading mutation, immune response and prognosis in endometrial cancer. Oncoimmunology 5(3):e1072675

36. Brnich SE et al (2019) Recommendations for application of the functional evidence PS3/BS3 criterion using the ACMG/AMP sequence variant interpretation framework. Genome Med 12(1):3

Publisher's Note Springer Nature remains neutral with regard to jurisdictional claims in published maps and institutional affiliations. 